\title{
Barrett-Dysplasie und -Frühkarzinom: Leitlinie aktualisiert
}

- In einem Delphi-Abstimmungsprozess, den man von der Entwicklung von S3-Leitlinien kennt, entwickelte eine große internationale Expertengruppe Empfehlungen zur Diagnostik und Therapie der Barrett-Dysplasie und des BarrettFrühkarzinoms. Die Experten kamen aus den USA, Japan und vielen europäischen Ländern. Insgesamt wurden 91 Aussagen getroffen, von denen einige wichtige im Folgenden wiedergegeben werden:

(1) Bei einem Barrett-Ösophagus sollten 4-Quadranten-Biopsien im Abstand von 1-2 cm sowie Biopsien aus allen auffälligen Arealen entnommen werden.

2 Die Diagnose einer Dysplasie sollte durch einen zweiten Pathologen bestätigt werden.

3 Bei hochgradiger Dysplasie oder T1m-Karzinom sollte eine endosko- pische Therapie (endoskopische Mukosaresektion EMR) in einem Zentrum erfolgen. Nach EMR sollte die verbleibende Barrett-Schleimhaut eradiziert werden. Die Radiofrequenzablation wird als die derzeit am besten verfügbare Methode zur Eradikation der Barrett-Schleimhaut angegeben.

4 Wenn eine hochgradige Dysplasie vorliegt, aber endoskopisch keine Läsion abgegrenzt werden kann, ist die Radiofrequenzablation die beste verfügbare Option. Bei Barrett-Frühkarzinom mit low-risk-Situation (Invasionstiefe $<500$ $\mu \mathrm{m}, \mathrm{G1-G2-Tumor,} \mathrm{keine} \mathrm{Lymphgefäß-}$ invasion) wird die endoskopische Behandlung als Alternative zur Operation gesehen.

\section{Kommentar}

\section{Testosteron macht Männer ehrlicher}

Testosteron fördert dominantes Verhalten und auch Aggressionen. Aber Testosteron macht auch ehrlicher, wie Experimente von Forschern an der Rheinischen Friedrich-Wilhelms-Universität Bonn ergeben haben.

- An dem Verhaltensexperiment nahmen 91 gesunde Männer teil. 46 von ihnen wurden mit Testosteron behandelt, indem das Hormon als Gel auf die Haut aufgetragen wurde. Als Vergleichsgruppe bekamen die anderen 45 Testpersonen lediglich ein Placebogel.

Alle Testpersonen führten ein einfaches Würfelspiel in separaten Kabinen durch. Je höher die gewürfelte Augenzahl, desto größer war der Geldbetrag, den es als Belohnung gab. Der Versuch war so konzipiert, dass die Probanden bei ihrer Eingabe in den Computer lügen konnten. Allerdings konnten die Wissenschaftler per Wahrscheinlich- keitsrechnung im Nachhinein feststellen, wie häufig in den Testgruppen durchschnittlich geschummelt worden war. Beim Würfelspiel logen Probanden mit erhöhtem Testosteronspiegel deutlich seltener $(<0,01)$ als jene, die nur ein Placebo erhalten hatten.

\section{Kommentar}

Dass die Probanden mit den höheren Testosteronwerten deutlich seltener logen als die unbehandelten Testpersonen, könnte daran liegen, dass das Hormon den Stolz und das Bedürfnis, ein positives Selbstbild zu entwickeln, steigert. Vor diesem Hintergrund reichten dann ein paar Euro, die man sich ermogeln konnte, als Anreiz nicht aus, um das Selbstwertgefühl aufs Spiel zu setzen.

K. MALberG =

\footnotetext{
- M. Wibral et al.

Testosterone administration reduces lying in men, Published on 10 Oct 2012, in PloSONE, DOI: 10.1371/journal.pone.0046774
}

Trotz der besseren Diagnostik mit hoch auflösenden Endoskopen werden weiterhin die 4-Quadranten-Biopsien alle 1-2 cm empfohlen. Leider geben die Autoren keine Empfehlungen über die Intervalle der Überwachungs-Endoskopien bei Barrett-Ösophagus. Aussagen zum Vorgehen bei niedriggradiger Dysplasie werden leider nicht getroffen. Die Autoren haben sich nach eigenen Aussagen auf die hochgradige Dysplasie und das Frühkarzinom fokussiert, da für diese Situationen die besten Evidenzen vorliegen. Die DGVS-Leitlinie von 2005 empfiehlt bei niedriggradiger Dysplasie in einer endoskopisch abgrenzbaren Läsion die endoskopische Abtragung, ansonsten Kontrollendoskopien (zunächst halbjährlich, dann jährlich).

M. GROSS =

\section{- C. Bennett et al.}

Consensus statements for management of Barrett's dysplasia and early-stage esophageal adenocarcinoma, based on a Delphi process. Gastoenterology 2012;143:336-346 